\title{
Configurations identitaires et contextes coloniaux
}

Une comparaison entre Tonga et Hawaii

\section{Paul Van Der Grijp}

\section{(2) OpenEdition Journals}

Édition électronique

URL : http://journals.openedition.org/jso/1601

DOI : 10.4000/jso. 1601

ISSN : $1760-7256$

Éditeur

Société des océanistes

Édition imprimée

Date de publication : 1 décembre 2001

Pagination : 177-192

ISSN : 0300-953x

Référence électronique

Paul Van Der Grijp, «Configurations identitaires et contextes coloniaux », Journal de la Société des Océanistes [En ligne], 113 | Année 2001-2, mis en ligne le 27 mai 2008, consulté le 21 avril 2019. URL http://journals.openedition.org/jso/1601 ; DOI : 10.4000/jso.1601 


\section{Configurations identitaires et contextes coloniaux : Une comparaison entre Tonga et Hawaii}

par

Paul VAN DER GRIJP *

\section{RÉSUMÉ}

Cet article tente une comparaison de certaines sociétés de la Polynésie occidentale et orientale en tant que configurations identitaires. Dans ces configurations, on doit prendre en compte non seulement les aspects idéels (valeurs, normes, etc.) mais également les aspects matériels comme, par exemple, la tenure foncière. Dans la mesure où l'on a affaire dans leur majorité à des sociétés rurales, l'identité englobe à la fois les idées que les gens ont dans leurs têtes et la (relation à la) terre qu'ils ont sous leurs pieds. Les changements dans ce domaine doivent être compris dans la perspective des contextes coloniaux, passés et contemporains.

Mots clés : identité culturelle, chefferie, tenure foncière, formes économiques, Polynésie.

Souvent, en anthropologie, les définitions des peuples non occidentaux se font en termes négatifs, à commencer par le mot non occidental : «ils» ne sont pas occidentaux. En plus, ils ne sont pas chrétiens, ils n'ont pas d'écriture, ils n'ont donc pas d'histoire (ils vivent dans la préhistoire, ils sont, comme le disait Eric Wolf (1982) des 'peoples without history'), ils sont non industriels, pré-capitalistes, non démocratiques, etc. En bref, ces peuples sont définis par ce qu'ils ne sont pas et par ce qu'ils n'ont pas. Il est sans doute temps de les caractériser en termes

\begin{abstract}
This article seeks to compare certain societies in western and eastern Polynesia as configurations of Polynesian identity. Within these configurations not only mental aspects are to be taken into account (values, norms, etc.) but also material aspects such as land ownership. Since we deal here merely with rural societies, identity comprises both the ideas people have in their heads and the (relation to the) land they have under their feet. Changes in the latter should be understood in terms of past and present colonial contexts.
\end{abstract}

Key words : cultural identity, chieftaincy, land tenure, economic forms, Polynesia

positifs, à partir de ce qu'ils ont et de ce qu'ils sont. C'est pour cela que je propose le concept de «configuration identitaire» que j'appliquerai dans une perspective comparative en Polynésie occidentale et orientale, en particulier dans les sociétés de Tonga et d'Hawaii. La version tongienne de l'identité polynésienne se compose d'un système socio-économique, politique et idéologique spécifique que je propose de ne pas réduire aux aspects idéels, mais de définir comme la configuration des quatre caractéristiques suivantes : (1) une chefferie et un système corres-

\footnotetext{
* Maître de conférences au Département d'ethnologie, Université de Provence (Université Aix-Marseille-I), et chercheur au Centre de Recherches et de Documentation sur l'Océanie (CREDO), Maison Asie Pacifique, Campus St. Charles, 3 Place Victor Hugo, 13003 Marseille (pvdgrijp@newsup.univ-mrs.fr).
} 


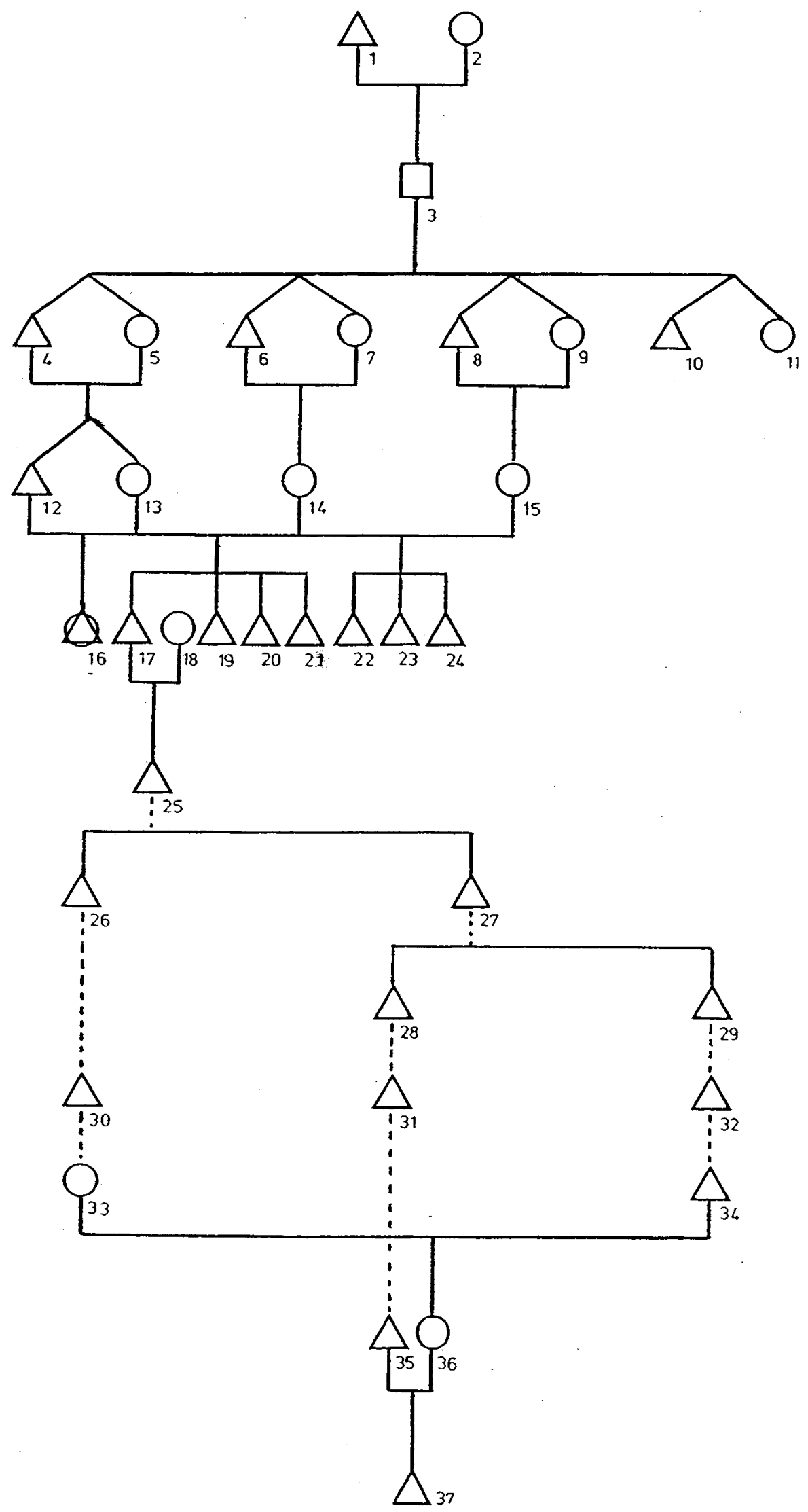

FIG. 1. - Les trois lignées de grands chefs, leur origine divine et la filiation royale contemporaine (les lignes pointillées indiquent le saut par-dessus plusieurs générations) 
pondant d'idéologie asymétrique fondé sur le complexe mana-tapu; (2) le rôle dominant de la parenté cognatique (avec un fort accent sur la relation frère - sœur) dans les relations sociales de production, de distribution, et de pouvoir ; (3) une forme de tenure foncière organisée par des principes relevant simultanément de la chefferie et de la parenté (cognatique) ; (4) une économie de subsistance, de troc et de dons.

Ce qui est typiquement tongien, par exemple, n'est pas la coexistence incidentelle de ces quatre caractéristiques, mais leur combinaison (configuration). Il ne s'agit donc pas de réduire l'identité tongienne uniquement à des valeurs et à des normes tongiennes (anga fakatonga), ou encore à la 'tradition' comme le fait, par exemple, Helen Morton quand elle parle de " grassroots understanding of tradition as it affects everyday life » (1996 : 21). Il ne faut pas non plus réduire l'identité tongienne à quelque chose qui serait un mode de production polynésien. L'identité tongienne possède à la fois des dimensions matérielles (par exemple la production des cochons, des tubercules, des denrées provenant de la mer, du kava, des nattes et du tapa) et des dimensions idéelles (des idées, valeurs, normes, etc.). Les dimensions matérielles et idéelles d'un état de société comme celui de Tonga sont aussi inséparables et complémentaires que les deux côtés d'une même feuille.

\section{L'identité tongienne comme une configuration de caractéristiques}

Au cours de l'histoire de Tonga, les quatre caractéristiques mentionnées ci-dessus se sont modifiées. Dès l'origine sans doute, la pratique et la pensée politique tongienne se trouvèrent conjuguées avec le religieux et la parenté. Avant les dernières guerres civiles, qui débutèrent autour de 1784, l'histoire socio-politique tongienne était dominée par les longues lignes de descendance des trois chefs suprêmes: Tu'i Tonga, Tu'i Ha'atakalaua, Tu'i Kanokupolu. La tradition orale fait remonter l'origine de la lignée $\mathrm{du}$ Tu'i Tonga au milieu du $\mathrm{x}^{\mathrm{e}}$ siècle, quand le premier Tu'i Tonga 'Aho'eitu ( $\mathrm{n}^{\mathrm{o}} 25$ dans la figure 1), naquit des amours du dieu Tangaloa et d'une mère terrestre (Reiter, 1907; Gifford, 1924 : 25-43 ; Bott, 1982 : 89-91). Tangaloa est une divinité connue dans tout le monde polynésien sous les noms de Takaroa à Tahiti, Tanaoa aux Marquises et Kanaloa à Hawaii (Tregear, 1891 : 463-464). À Tonga, il y avait quatre Tangaloa ( ${ }^{\circ}{ }^{\circ} 17,19,20$ et 21$)$. 'Aho'eitu était le fils de l'aîné des Tangaloa, 'Eitumatupu'a ( ${ }^{\circ}$ 17). Dans le panthéon tongien, les Tangaloa, leurs trois frères appelés Maui $\left(\mathrm{n}^{\circ} 22,23\right.$ et 24$)$ ainsi que leur germain aîné, la divinité bisexuelle Hikuleo ( $\left.\mathrm{n}^{\circ} 16\right)$, constituent la cinquième génération de la généalogie originaire ( $c f$. DouaireMarsaudon, 1998 : 269-272). Au travers de cette généalogie, c'est la force surnaturelle ou le mana, et la puissance d'imposer les prohibitions ou tapu (tabous), qui sont transmis au premier Tu'i Tonga ainsi qu'à ses descendants directs. On est ici devant la première des caractéristiques de l'identité tongienne, telle qu'elle a été définie ci-dessus : une chefferie suprême et le système correspondant d'idéologie asymétrique basée sur le complexe mana-tapu.

À l'origine, le Tu'i Tonga était un chef à la fois sacré et profane qui ne garantissait pas seulement symboliquement - par des mythes et des rites - le bien-être de toute la population tongienne mais qui possédait aussi un pouvoir militaire et politique considérable. Au cours du $\mathrm{Xv}^{\mathrm{e}}$ siècle, les Tu'i Tonga auraient ainsi étendu leur hégémonie jusqu'à Niue, Samoa, Tokelau, Wallis, Futuna, Rotuma, et sur une partie du groupe Lau à Fidji (Latukefu, 1975 : 2). Selon la tradition orale, cependant, cette expansion de l'empire tongien souleva de tels problèmes politiques que le $24^{\mathrm{e}}$ Tu'i Tonga, Kau'ulufonua ( $\left.{ }^{\circ} 26\right)$, décida de déléguer les tâches profanes du gouvernement à son frère cadet, tandis que luimême continuait d'assumer les fonctions religieuses de la charge de chef suprême (voir Herda, 1988 ; Mahina, 1992). Toujours selon la tradition orale, ce frère cadet, Mo'ungamotua $\left(\mathrm{n}^{\circ} 27\right)$, devint le premier représentant d'un nouveau titre, le Tu'i Ha'atakalaua. Le sixième Tu'i Ha'atakalaua, Mo'ungatonga, créa également pour un de ses fils, Ngata ( $\left.{ }^{\circ} 29\right)$, un nouveau titre, Tu'i Kanokupolu, en lui attribuant les responsabilités militaires et politiques du gouvernement à Hihifo. Mo'ungatonga conservait quant à lui le pouvoir nominal, dont hérita plus tard son fils aîné Fotofili $\left(n^{\circ} 28\right)$ et les descendants de celui-ci. Aux XVI ${ }^{\mathrm{e}}$ et $\mathrm{XVII}^{\mathrm{e}}$ siècles, le climat politique paraît avoir été assez stable (Latukefu, 1974 : 9). En 1643, le voyageur néerlandais Abel Tasman remarquait que les Tongiens ne portaient aucune arme (1643: 62). À l'époque du contact, la société tongienne était très stratifiée. De haut en bas, la pyramide socio-politique était constituée des « états » ou des « ordres » suivants : les chefs suprêmes ou ha'a tu'i, les chefs ordinaires ou hou'eiki, les maîtres de cérémonies des chefs ou kau matapule, les descendants et frères cadets des matapule ou kau mu'a, les roturiers ou kau tu'a, et les 'esclaves', probablement des prison- 
niers de guerre, ou kau popula (Martin, 1817 : 290-297 ; Gifford, 1929 : 111) ${ }^{1}$.

Le triumvirat des trois chefs suprêmes ne devait pas survivre à la guerre civile et à son cortège d'intrigues politiques, de meurtres, et de batailles auxquelles participaient des centaines et parfois des milliers de Tongiens. En 1799, le seizième Tu'i Ha'atakalaua, Mulikiha'amea $\left(\mathrm{n}^{\mathrm{o}} 31\right)$, fut tué sur le champ de bataille. Personne désormais n'occupera plus la fonction de Tu'i Ha'atakalaua, devenue vacante. En 1826, l'armée de Laufilitonga, qui devenait Tu'i Tonga plus tard $\left(\mathrm{n}^{\circ} 30\right)$, fut battue par l'armée de Taufa'ahau $\left(\mathrm{n}^{\circ} 32\right)$, un grand chef qui se fera attribuer le titre de Tu'i Kanokupolu au cours de sa carrière politique. Après sa victoire militaire, Taufa'ahau toléra l'existence du chef suprême, le Tu'i Tonga Laufilitonga - dont la puissance était sacrée - jusqu'à sa mort en 1865 , mais empêcha tout autre personne de reprendre le titre. Au cours de la guerre civile, Taufa'ahau, avec l'aide d'armes à feu et le support moral des missionnaires européens et de la marine britannique, réussit à s'emparer du pouvoir politique et fit de Tonga un État centralisé. Suivant le modèle royal occidental, plus précisément britannique, Taufa'ahau fut couronné roi de Tonga en 1845 (Van Der Grijp, 1992, 1993c). Au cours des décennies suivantes, son autorité fut renforcée par plusieurs recueils de lois écrites, puis par une constitution (en 1875). Cette transformation d'une chefferie suprême en un système de royauté constitutionnelle se révéla vitale: elle permit au nouvel État tongien d'être reconnu internationalement, constituant ainsi une précondition pour son autonomie relative (Campbell, 1992 ; Rutherford, 1971 ; Van Der Grijp, 1993d). Suivant en cela une politique matrimoniale inaugurée au $\mathrm{XVII}^{\mathrm{e}}$ siècle, la Reine Salote Tupou III ( $\mathrm{n}^{\mathrm{o}} 36$ ), mère du roi actuel, se maria avec un descendant du lignage de Tu'i Ha'atakalaua, Viliami Tungi Mailefihi $\left(\mathrm{n}^{\mathrm{o}} 35\right)$; son père, le Roi Siaosi Tupou II $\left(\mathrm{n}^{\circ} 34\right)$, s'était marié lui-même avec Lavinia $\left(\mathrm{n}^{\circ} 33\right)$, une descendante de la dynastie du Tu'i Tonga. Grâce à ces unions royales, les trois lignages des chefs suprêmes se trouvaient converger - au moins dans l'idéologie de la maison royale - en la personne du roi actuel, Taufa'ahau Tupou IV (n $\left.\mathrm{n}^{\mathrm{o}} 37\right)$.
À la fin $\mathrm{du} \mathrm{XIX}^{\mathrm{e}}$ et au début $\mathrm{du} \mathrm{Xx}^{\mathrm{e}}$ siècle, certains chefs obtinrent des titres nobles (nopele en tongien) pourvus de domaines fonciers. Ceux-ci constituaient des possessions inaliénables (tofi'a) liées aux titres de la noblesse et transmissibles selon les principes de patrilinéarité et de primogéniture. La plus grande partie des terres cultivables se trouvait aux mains de ces nobles. En dehors de la famille royale, ces nobles ont été, depuis la Constitution, la source la plus importante du pouvoir dans la politique tongienne. Actuellement, il existe 33 titres de noblesse et les rangs hiérarchiques sont toujours considérés comme «le concept le plus présent dans la culture tongienne » (Kaeppler, 1971: 174) ${ }^{2}$. Récemment, l'influence et le pouvoir de l'aristocratie tongienne ont été mis en cause par la nouvelle classe moyenne, en particulier celle des enseignants et des entrepreneurs (Benguigui, 1989 ; James, 1994, 1998 ; Latukefu, 1994 ; Lawson, 1996).

L'organisation cognatique de la parenté et son articulation autour de la paire frère-sœur - ce que j'ai défini comme la deuxième caractéristique de la version tongienne de l'identité polynésienne - joue toujours un rôle important dans les relations de production, de distribution et de pouvoir. Aujourd'hui, cependant, le rôle de la parenté se trouve en compétition avec le management de style occidental, le marketing et les principes inspirés par le modèle nouveau de la " démocratie ». La dichotomie tu'a-'eiki (inférieur-supérieur) exprime la distinction roturierschefs, mais aussi une inégalité sociale pertinente au sein du domaine de la parenté. En effet, dans la parenté, un Tongien peut être soit tu'a (de statut inférieur) soit 'eiki (de statut supérieur) vis-à-vis de tout autre Tongien : (1) les sœurs ont un statut supérieur aux frères quel que soit leur âge respectif ; (2) les germains aînés ont un statut supérieur aux germains cadets ; (3) dans la génération antérieure à ego, la parentèle patrilatérale (en particulier la sœur aînée du père, la mehekitanga) a un statut supérieur à ego, et la parentèle matrilatérale (en particulier le frère de la mère, le tu'asina) un statut inférieur ${ }^{3}$; (4) les enfants de la sœur de ego ont un statut supérieur à ego si ego est masculin. Ces différences de statuts correspondent à un ensemble de normes (listées et illustrées ethnographiquement dans Van Der

1. Dans ce contexte, les notions de ha' $a$, hou et kau indiquent une catégorie sociale ou classe.

2. Il y a cependant quelque anicroche à cette vision des choses, comme en témoignent les propos du savant tongien 'Epeli Hau'ofa : «We still expect to see in our aristocracy, as in no other group in our society, the ideal qualities of our collective personality. In our hurly-burly, free-for-all, dog-eat-dog modern society, we look to them for such qualities in social interaction as civility, graciousness, kindness, and that calming aura of a unifying presence in our midst. This may explain why we get very disappointed whenever they behave as mere mortals, exhibiting the follies and foibles that are the lot of humanity in general » (1994: 427).

3. Le statut des oncles et des tantes de ego s'étend à leurs enfants, quel que soit leur âge ou leur sexe. 
Grijp, 1993b : chap. IX). La plus importante est le respect et l'évitement entre germains (et par extension entre cousins) de sexe opposé ${ }^{4}$. Helen Morton observe qu'aujourd'hui, "dans plusieurs familles les tabous frère-sœur ne sont pas toujours respectés, et même des gens âgés précisent quelquefois que leurs relations avec des germains de l'autre sexe pendant leur jeunesse étaient faingofua» (1996: 133), c'est-à-dire facile et simple. Cet « affaiblissement de la relation d'évitement entre frères et sœurs » est fortement désapprouvé par beaucoup de Tongiens (ibid., 135). Cependant, la relation frère-sœur ne peut pas être réduite à l'évitement et à des restrictions (faka'apa'apa): elle est également caractérisée par un support mutuel et, en particulier, par l'assistance économique des frères envers les sœurs et les enfants de celles-ci ${ }^{5}$. Les aspects symboliques ou idéels (valeurs et normes) et les pratiques matérielles (par exemple, économique) peuvent être distingués analytiquement mais ne le sont pas dans la réalité sociale. Ainsi, la dichotomie tu'a-'eiki est une question de logique relationnelle, laquelle, comme l'a bien vu Brad Shore, correspond à une " perspective épistémologique polynésienne plus générale selon laquelle les choses sont appréhendées dans leurs contextes spécifiques et au travers de leurs effets perçus dans le monde plutôt qu'en terme de traits essentiels, intrinsèques » (Shore, 1989 : 138) ${ }^{6}$. Le comportement des personnes de bas statut est incorporé dans les valeurs culturelles d'amour et d'attention ('ofa), de respect (faka'apa'apa), et d'obéissance (talangofua) (Morton, 1996 : 70).

Nous avons défini la troisième caractéristique de la version tongienne de l'identité polynésienne comme une forme de tenure foncière structurée par les principes de la chefferie et de la parenté cognatique. Les nobles, qui sont apanagés ou, si l'on préfère, pourvus de domaines, sont tenus par la loi de distribuer la plus grande partie de leur domaine à leurs tenanciers. Chaque Ton- gien, âgé de seize ans ou plus, a un droit légal (constitutionnel) à l'usufruit d'une parcelle de terre agricole ('api 'uta) comprenant 8,25 acres (c'est-à-dire 3,34 hectares) et d'une parcelle plus petite ('api kolo) de 0,4 acre (ou 0,16 hectare) dans un village ou une ville où il peut construire une maison ${ }^{7}$. Le droit à l'usufruit de ces deux parcelles se transmet du père au fils aîné. À Tonga, personne, officiellement, n'a la permission de vendre la terre - ni les utilisateurs directs, ni les nobles, ni même le roi. Comme il est écrit dans la constitution : "Il ne sera jamais légal pour personne dans ce pays [...] de vendre la moindre parcelle de la terre du royaume de Tonga » (cité dans Latukefu, 1974 : 278 ; voir aussi Latukefu, 1975, 1997 ; Powles, 1990, 1993). L'économie tongienne étant surtout basée sur l'agriculture, la terre reste le moyen de production principal. Depuis plusieurs décennies, cependant, le manque de terre est de plus en plus important, comme le montrent les statistiques gouvernementales. Cependant, de telles statistiques sont trompeuses, comme cela a pu être observé dans une étude des terres villageoises sur l'île de Tongatapu en 1983 :

« Only about one-third of 'Olungans were landholder, but two-thirds of the adult population belonged to a household that had land. What is more, the majority of those households without any landholder had access to land or its produce through other relatives [...]. Although the great majority of 'Olungans held neither registered, nor unregistered land, few villagers were totally without access to land or produce » (Small, 1997 : 28).

Ici, la troisième caractéristique de la version tongienne de l'identité polynésienne rejoint la seconde caractéristique (le rôle dominant de la parenté cognatique) : l'accès à la terre distribuée par des réseaux de parenté. Récemment, il y a des exemples de vente de terres, souvent sous le couvert de dons « traditionnels ", me' $a$ 'ofa ( $c f$. Van Der Grijp, 1993a). Le développement de

4. Dans le mythe de l'origine du monde et des divinités, l'inceste frère — sœur est une règle, comme on peut le voir dans la troisième et la quatrième génération de la figure 1. Voir aussi, pour les rapports tongiens frère-sœur Hocart (1915), RadcliffeBrown (1924), Rogers (1977), Helu (1995, 1999) et Douaire-Marsaudon (1998).

5. Voir aussi les exemples donnés par Françoise Douaire-Marsaudon (1998 : 149-150).

6. Dans son étude sur la socialisation des enfants, Morton met l'accent sur la centralité de cette dichotomie pour les notions tongiennes d'identité et de personnalité : « Children occupy one of the lowest status positions in Tongan society and must learn the values and behaviors appropriate to this status. At the same time, chiefly characteristics are upheld to them as highly valued... [At an early age, children learn] to use a respectful stance and tone when addressing higher-status persons, and the mixture of deference and fear they exhibit on such occasions contrasts markedly with the aggressive or whining demands they make of their peers and juniors... Tu' $a$ children are also provided with clear models of deferential and submissive behavior by their older siblings and adult kin within the context of these relatives' interactions with persons of higher rank or status » (1996:25, 90, 95).

7. À Ha'apai et dans d'autres parties de Tonga, les parcelles individuelles des paysans sont plus petites. Sur la petite île de Kotu, dans la partie occidentale de Ha'apai (district Lulunga), par exemple, les parcelles ont en moyenne 0,9 acre, et varient entre 0,4 et 2,5 acre. Cependant, $80 \%$ des 35 unités domestiques de Kotu ont accès aux terres de l'île, volcanique et plus large, de Tofua (Perminow, 1993 : 8 ; voir aussi Van der Grijp, 1993b : 80-81, 91-94). 
l'exportation du potiron au Japon augmente la valeur commerciale de la terre et, comme conséquence, la vente illégale de la terre est de plus en plus pratiquée. Le résultat est idéologiquement enrobé dans un discours de circulation de dons « traditionnels ».

La quatrième caractéristique de la version tongienne de l'identité polynésienne est la combinaison d'une économie de subsistance et de dons. Ces dons consistent principalement en cochons, en tubercules, en denrées provenant de la mer, en kava, en nattes et en tapa. Autrefois, les Tongiens avaient l'habitude d'élever des cochons, des chiens et des poules. Dans l'histoire plus récente, s'y sont ajoutés des chevaux, des vaches et des chèvres. De tous ces animaux, les cochons sont les plus valorisés. Comme en Mélanésie ( $c f$. Dwyer, 1990 ; Lemonnier, 1993 ; Rappaport, 1968), les cochons ne sont pas élevés pour la consommation quotidienne, mais sont abattus en masse pour des grandes cérémonies de dons. La nourriture à base d'amidon comme par exemple les tubercules (l'igname, le taro, le taro " géant ", les patates douces et le manioc), les bananes et les arbres à pain fournissent l'essentiel de l'alimentation quotidienne, tandis que la mer (tahi) apporte les protéines. Sur les récifs et dans le lagon, les femmes tongiennes, souvent assistées par leurs enfants, collectent ce qui est regroupé sous le terme de fingota: les algues, les mollusques, les holothuries, les oursins, les poulpes, les seiches, les crustacés et les petits poissons ( $c f$. Bidesi, 1994 ; Malm, 1999 ; Matthews, 1995). Les plus gros poissons comme les cétacés, les raies et les tortues sont pêchés par les hommes en haute mer (moana) (BatailleBenguigui, 1994: 110-111). Parmi toutes les catégories de nourriture, la viande de porc est celle qui est le plus valorisée culturellement, suivie de près par les plus grandes ignames, les kahokaho. Le kava est la boisson cérémonielle et sociale de Tonga ( $c f$. Biersack, 1991 ; DouaireMarsaudon, 2001). Il est fait avec de la poudre de racines séchées et pilées de l'arbuste de kava (Piper methysticum), mélangée avec de l'eau dans une coupe en bois $\left(\operatorname{tano}^{\prime} a\right)$. On distingue différentes situations sociales dans lesquelles les hommes boivent le kava : la cérémonie du kava royal, celle du kava noble et celle du kava informel ou des roturiers (taumafa kava, 'ilo kava et faikava respectivement). Le tapa et les nattes fines sont fabriqués, présentés et échangés par des femmes ; conçus comme le 'trésor' (koloa) des femmes, ces objets sont d'une importance suprême dans nombre de rituels et dans la circu- lation des dons ( $c f$. Van der Grijp, 1993e; Douaire-Marsaudon, 1996 ; James, 1997; Kaeppler, 1999) ${ }^{8}$.

Le récent développement du potiron à Tonga est un bon marqueur de la transition d'une agriculture de subsistance à une agriculture commerciale, et c'est aussi l'indicateur d'une accélération pertinente dans ce processus. Cependant, les produits agricoles commerciaux existaient déjà dans la dernière partie du $\mathrm{XIX}^{\mathrm{e}}$ siècle (huile de coco, coprah, coton) et continuaient de jouer un rôle dans l'histoire agricole de Tonga (bananes, pastèques, vanille, etc.). Aujourd'hui, l'agriculture de Tonga peut toujours être caractérisée comme la combinaison d'une agriculture de subsistance et d'une agriculture commerciale, et son économie comme la combinaison d'une économie de subsistance / dons et d'une économie monétaire, quatrième caractéristique de la version tongienne de l'identité polynésienne. L'économie de Tonga est, comme l'exprimait Chris Morgan, « a changing conjunction of a developing commodity mode with a gift complex premised on island concepts of reciprocity within hierarchy and inequality $(1985: 160)$. Aujourd'hui, l'État tongien ne peut être vu ni comme capitaliste ni comme purement polynésien, mais il constitue, dans sa structure de production et de pouvoir, un mélange unique d'aspects capitalistes et de traits polynésiens. Bien que le développement récent du potiron ne soit pas le premier pas vers le capitalisme, il en constitue cependant un facteur d'accélération évident. Pourtant, ceci n'implique pas que Tonga puisse un jour perdre complètement son caractère polynésien et devenir strictement capitaliste ( $c f$. la définition ci-dessous). Pour comprendre la combinaison tongienne singulière $\mathrm{du}$ capitalisme et de l'identité polynésienne, il est essentiel d'analyser les phénomènes contemporains des booms de la vanille et du potiron dans leurs implications à la fois socio-économiques et culturelles.

Tonga n'a jamais été colonisé directement par un système ou gouvernement étranger. $\mathrm{Au}$ milieu du XIX ${ }^{\mathrm{e}}$ siècle, Tonga passa d'une situation dans laquelle plusieurs groupes de chefs dominaient les îles à un pouvoir d'État centralisé avec un roi : une chefferie-État moderne (Van Der Grijp, 1993d). Aujourd'hui, Tonga est un état moderne avec toutes les institutions habituelles : un gouvernement avec un parlement, des fonctionnaires, des lois, des juges, une police, une armée, des écoles, un système de santé, etc. Simultanément, cependant, Tonga possède plu-

8. Les nattes sont faites de feuilles de palme de pandanus et de cocotier et le tapa provient de l'écorce du mûrier à papier (hiapo). 
sieurs caractéristiques d'une chefferie polynésienne, mais dans des formes " adaptées » à la modernité. C'est cet ensemble de traits polynésiens dits « traditionnels » et de caractéristiques dites « modernes » qui constitue l'identité tongienne actuelle.

\section{L'entreprenariat et la genèse d'une nouvelle classe moyenne}

L'introduction récente des cultures commerciales comme la vanille et le potiron a eu un impact socio-économique important sur les petits cultivateurs et a également accéléré la genèse d'un entreprenariat agricole, chargé de la production et de l'exportation de produits agricoles commerciaux (Van der Grijp, 1997a, 1997b, 1999). La distinction entre entrepreneurs et petits cultivateurs dans l'agriculture commerciale de Tonga pourrait être interprétée comme une des formes de la dichotomie marxiste du XIX ${ }^{\mathrm{e}}$ siècle, capitalistes versus prolétariat. Sans nier le fait que ces catégories sociales existent encore en Europe, ou même à Tonga, la situation vers la fin $\mathrm{du} \mathrm{Xx}^{\mathrm{e}}$ et au début du XXI ${ }^{\mathrm{e}}$ siècle est plus complexe que ce que suggère cette dichotomie. Même en Europe occidentale, en Amérique du Nord et dans les pays asiatiques hautement capitalistes, les "propriétaires" des entreprises privées ne sont souvent pas complètement - parfois ni même partiellement - les propriétaires des moyens de production dans leurs entreprises respectives. Les vrais propriétaires peuvent être des banques ou d'autres grandes institutions d'investissement, comme par exemple les sociétés d'assurances. Les marchandises vendues dans les magasins n'appartiennent généralement pas au propriétaire du magasin, mais aux fournisseurs principaux ; le bâtiment du magasin est d'habitude loué; d'autres investissements de l'affaire sont rendus possibles par des emprunts bancaires. Les employés d'une chaîne de production peuvent être des actionnaires de la société privée pour laquelle ils travaillent. Et on pourrait développer la question avec le phénomène relativement récent des classes moyennes qui sont toujours en expansion. La riche variété des formes intermédiaires entre capitalistes et prolétaires existe aussi dans le secteur monétarisé de l'économie tongienne actuelle. De plus, à Tonga, le principal moyen de production agricole, c'està-dire la terre, ne peut jamais formellement (selon la constitution) devenir propriété privée puisque la terre ne peut être vendue. Qu'est-ce donc qu'un entrepreneur? Est-il, ou non, un capitaliste ? Et qu'en est-il à Tonga ?

Les entrepreneurs peuvent être considérés comme des représentants d'une configuration socio-économique spécifique, le capitalisme. En anthropologie, un entrepreneur est souvent défini comme une personne qui utilise et stimule des innovations technologiques et qui mobilise le travail et le capital par un projet industriel ou commercial avec le but de faire du profit ( $c f$. Barth, 1966 : 17-18). Dans la perspective adoptée ici, les entrepreneurs sont conçus comme des acteurs sociaux, agissant dans le contexte du mode de production capitaliste. Nous définirons le capitalisme comme la configuration suivante : production de marchandises, propriété privée des moyens de production, utilisation de l'argent pour 'faire' plus d'argent et, finalement, travail salarié. Dans cette configuration, les moyens de production et l'argent fonctionnent comme un capital. L'argent, dans ce sens, n'est pas seulement liquide, donc une chose physique, mais inclut, entre autre chose, du crédit 9 . Par l'exploitation du travail salarié, le capital est utilisé pour produire de la plus-value, c'est-à-dire une valeur qui excède la valeur originelle dans le processus de production, et donc accumuler du capital d'où le mot capitalisme (Godelier, 1991 ; Van Der Grijp, 1993b : 4-6). À Tonga autrefois, comme dans d'autres sociétés polynésiennes, aucune de ces caractéristiques n'existait. À Tonga aujourd'hui, avec son entreprenariat local en progression, plusieurs de ces caractéristiques coexistent, mais elles ne forment pas une unité cohérente ${ }^{10}$. Elles sont en effet trop mêlées avec la version tongienne de l'identité polynésienne ${ }^{11}$. Un développement, intimement lié à celui dont on vient de parler, est la genèse d'une classe moyenne ${ }^{12}$. À Tonga, la nouvelle classe

9. Dans les sociétés capitalistes, 'the quantity of money is the resultant of many forces including the velocity of its circulation... [Also] stocks and shares, mortgages, grain futures, real property [may] take on the functions of money' (Belshaw, $1965: 9-10)$.

10. Le mot tongien pour capitalisme est fakapa'anga, littéralement : à la façon de l'argent.

11. Voir pour d'autres perspectives sur les entrepreneurs à Tonga, Brown and Connell (1993), Ramanlal (1990) et Ritterbush (1988) ; pour des études sur l'identité polynésienne liée avec l'économie locale à Samoa : Macpherson (1988), O'Meara (1990), Tcherkézoff (1992), Tiffany (1975) ; pour Fidji : Abramson (1999), Belshaw (1964), Fisk and Honeybone (1971); et pour Rotuma : Howard (1996) et Rensel (1994).

12. Le concept de classe moyenne peut être retracé en Europe jusqu'en 1785, quand Thomas Gisborne l'utilisait pour indiquer « la classe des propriétaires et des entrepreneurs entre les propriétaires fonciers d'un côté et les travailleurs agricoles et 
moyenne est composée de Tongiens éduqués et salariés mais aussi d'entrepreneurs tongiens.

\section{Continuité et discontinuité dans la «culture traditionnelle ".}

Le fait que Tonga n'ait jamais été colonisé et garde toujours les caractéristiques-clé de son identité polynésienne, lui donne une position assez unique dans le débat concernant l'invention de la tradition ${ }^{13}$. Il est intéressant de comparer sous cet angle Tonga avec Samoa, également en Polynésie occidentale. Dans leur livre Samoan Village: Then and Now (1992), Lowell Holmes et Ellen Rhoads Holmes ont posé une question fondamentale : " pourquoi la société de Samoa a-t-elle été capable de conserver autant sa culture traditionnelle ? ». Selon eux la réponse se trouve " dans la nature de la culture même, en particulier au plan des attitudes et des institutions vis-à-vis de la famille, du gouvernement et du surnaturel » (1992 : 100). Leur argument est que "dans la plupart des situations où deux cultures se rencontrent, le changement culturel sera en général plus grand dans des sociétés où le système traditionnel récompense un plus petit nombre de gens. Si un système nouveau plus égalitaire se présente, la majorité de gens auront moins tendance à s'engager dans un système qui ne récompense qu'un petit nombre de gens " (ibid.). Holmes et Holmes opposent Samoa à Tahiti, à la Nouvelle Zélande, aux îles Cook, aux Marquises, à Hawaii, aux Tuamotus, "régions où l'acculturation a été marquée ». Selon ces auteurs, dans toutes ces sociétés, en contraste avec Samoa, « les cultures ont accentué l'importance de la primogéniture, les lignages aînés, et d'autres formes de stratification. De plus, les élites qui ont profité de ces arrangements furent certainement encouragées dans leurs positions par des sanctions religieuses sous forme de mana et tapu. Il est probable que la majorité de la population n'ait pas apprécié la situation dans laquelle quelques uns seulement ont eu le plaisir du pouvoir et du prestige, mais il était difficile de s'opposer à un système qui avait la bénédiction du surnaturel » (ibid.).

Une telle argumentation est fort discutable. Stratifiée socialement comme l'étaient Tahiti et Hawaii, Tonga n'est pourtant pas du tout acculturée au même degré que Tahiti et Hawaii ou que les autres sociétés polynésiennes mentionnées par Holmes et Holmes. En termes de degrés d'acculturation, Tonga appartient plutôt à la même catégorie que Samoa ${ }^{14}$. Dans la littérature comparative sur la Polynésie (Burrows, 1939, 1940 ; Sahlins, 1958 ; Goldman, 1970), Tonga est présentée, aux côtés de Tahiti et de Hawaii, comme une des sociétés polynésiennes les plus stratifiées. Bien que les textes classiques de Burrows, Sahlins et Goldman aient reçu beaucoup de critiques sur d'autres aspects, ils ont toujours autorité sur ce point là ( $c f$. Howard et Borofsky, 1989). Donc, l'argumentation « une forte stratification sociale $\rightarrow$ " plus de changements culturels » ne tient pas. Il vaut mieux chercher la réponse à la question de Holmes et Holmes sur la continuité et la discontinuité dans la "culture traditionnelle» dans la forme et l'intensité de la colonisation étrangère (occidentale) aux $\mathrm{XIX}^{\mathrm{e}}$ et $\mathrm{Xx}^{\mathrm{e}}$ siècles plutôt que dans le degré de la hiérarchisation des sociétés concernées. En ce qui concerne les aspects politiques, économiques et démographiques, cette colonisation fut plus forte à Hawaii, en Nouvelle Zélande et dans les îles de (ce qui est appelé maintenant) la Polynésie française qu'à Tonga et Samoa. Tonga, qui est une des sociétés polynésiennes les moins "acculturées», peut être opposée à Hawaii, laquelle se situe — avec celle des Maoris de la Nouvelle Zélande - à l'autre extrémité de l'échelle d'acculturation polynésienne. Dans la section suivante, nous allons comparer Tonga et Hawaii à partir des quatre caractéristiques que nous avons identifiées précédemment comme formant la configuration (tongienne) de l'identité polynésienne.

urbains de l'autre ». Au XIX ${ }^{\mathrm{e}}$ siècle, on a continué à utiliser ce concept avec la même signification. Au Xx ${ }^{\mathrm{e}}$ siècle, le terme de « classe moyenne» s'étend aux professions dites des cols blancs, allant des emplois administratifs peu qualifiés, jusqu'aux médecins, avocats, et universitaires. En général, son usage est limité aux employés non-manuels, à l'exclusion des propriétaires des moyens de production, comme les usines et la terre. $\mathrm{Au}$ cours du $\mathrm{xx}^{\mathrm{e}}$ siècle, la proportion des employés non manuels augmenta considérablement. En Grande Bretagne, par exemple, de $19 \%$ en 1911 à $47 \%$ en 1981 (Urry, $1993: 381$ ). La genèse de la classe moyenne est générée par le capitalisme : pour le marxisme, c'est en raison des relations sociales de la production, pour les théoriciens weberiens, en raison des mécanismes du marché. Depuis les années 1980 et 1990 et au début du XXI siècle, à cause de l'intérêt de plus en plus fort pour les processus de mondialisation - à la fois dans la production et la circulation cette opposition théorique entre marxistes et weberiens a tendance à devenir moins marquée.

13. Dans sa forme actuelle, le débat a été lancé par les ouvrages édités par Keesing and Tonkinson (1982) et Hobsbawm and Ranger (1983); voir aussi les volumes édités par Van der Grijp et Van Meijl (1993, 1994), Otto et Thomas (1997), et Babadzan (1999).

14. Rappelons que, après son introduction dans les années 1930, le concept d'acculturation a été un point focal du débat anthropologique dans les années 1950, quand Lowell Holmes a fait son premier terrain à Samoa. 


\section{Configurations identitaires à Tonga et à Hawaii}

La première caractéristique de la version tongienne de l'identité polynésienne a été définie par l'existence d'une chefferie et d'un système correspondant d'idéologie asymétrique (la dichotomie tu'a-'eiki) fondée sur le complexe mana - tapu. Partout en Polynésie, les chefs suprêmes descendaient en ligne directe des dieux « ce qui donnait aux premiers le droit au respect et aux privilèges selon la coutume donnés aux derniers comme des formes de vénération; comparés aux autres gens, de tels chefs sont 'divins' et 'sacrés' » (Sahlins, 1995 : 252). Au XIX ${ }^{\mathrm{e}}$ siècle, à Tonga et à Hawaii, la chefferie suprême fut transformée en un système de monarchie constitutionnelle, accompagné, à Tonga, de la création d'une noblesse foncière. À Hawaii, et dans des conditions assez semblables à celles de Tonga, après le contact avec l'Occident et au moyen des armes à feux et des conseils militaires occidentaux, un chef de haute naissance, Kamehameha I, se montra capable d'établir et de maintenir une hégémonie militaire sur l'ensemble de l'archipel (et non plus sur une seule île). Mais, dans le processus de colonisation, Hawaii perdit simultanément son système de chefferie et les structures d'autorité indigène qui lui étaient associées. Le cas diffère donc passablement de celui de Tonga.

La deuxième caractéristique de la version tongienne de l'identité polynésienne est constituée par le rôle dominant de la parenté (cognatique) dans les relations sociales de production, de distribution, et de pouvoir. On a également mentionné le fort accent sur la relation frère-sœur, mais ceci est spécifique pour la Polynésie occidentale et moins accentué en Polynésie de l'Est, dont Hawaii. On a vu que malgré le fait que la parenté tongienne y soit en compétition avec le management, le marketing et les valeurs de la démocratie à l'Occidentale, la parenté est toujours un élément crucial dans les relations sociales de production, de distribution et de pouvoir. À Hawaii, selon Jocelyn Linnekin, « l'organisation sociale des roturiers est à la fois rattachée au système de chefferie et complémentaire de ce dernier » (1990 : 113). Dans le passé, c'est-à-dire avant qu'ils aient complètement perdu leur pouvoir, "les chefs n'étaient pas du peuple, ils n'étaient pas à la tête des lignages locaux » (ibid. 152). Sahlins a fait une remarque similaire: «bien que vrai pour toute la Polynésie, le lien entre le chef et les dieux avait un impact plus radical à Hawaii en raison d'une discontinuité généalogique entre ces êtres supérieurs et les couches inférieures de la population » (1995: 128).

Pour notre comparaison avec Hawaii, nous avons choisi le village de Keanae sur l'île hawaiienne de Maui, où l'anthropologue Jocelyn Linnekin a travaillé. Ce village est aujourd'hui " une communauté américaine moderne, intégrée dans l'économie de marché et participant aux distinctions sociales et au développement politique dans la société en général » (Linnekin, 1985 : 1). Cette société « en général » est celle des États-Unis. Linnekin : « les villageois de Keanae sont des Hawaiiens-Américains, qui participent dans les institutions politiques et économiques nationales (autrement dit des États-Unis), mais ils ont aussi un héritage non occidental $»$ (ibid. 4). Le concept clé de cet héritage est celui de 'ohana, qui désigne la famille étendue. Bien que ce concept de 'ohana ait été décrit dans les rapports sur la société hawaiienne d'autrefois, ce concept « apparaît rarement dans des textes et matériaux d'archives du $\mathrm{XIX}^{\mathrm{e}}$ siècle, mais aujourd'hui il réfère à une version idéalisée de l'unité de la famille hawaiienne » (ibid. 12). Cette famille étendue, qui ne joue aujourd'hui presque aucun rôle dans les relations sociales de production et de pouvoir politique, prend une place fondamentale dans la préparation de la nourriture pendant les grands festins, luau, à l'occasion des naissances, des anniversaires et des mariages. Les luau sont des marqueurs d'appartenance et l'idéal de aloha constitue la variante hawaiienne $\mathrm{du}$ 'ofa tongien (affection, compassion, attention, générosité, amour). À l'origine dédiés aux anciens dieux, « la nourriture et le travail pour le luau étaient les présents suprêmes échangées entre les Hawaiiens » (ibid. 115). Si, dans ces derniers contextes, la parenté hawaiienne représente un enjeu important, son rôle économique et politique est cependant assez limité, comparé à celui de la parenté tongienne contemporaine. Ceci est bien évidemment lié au fait que, pour les Tongiens, la « société en général » n'est pas une entité (pseudo-)coloniale étrangère, mais est toujours la société tongienne.

La troisième caractéristique de la version tongienne de l'identité polynésienne correspond à une forme de tenure foncière structurée par des principes de chefferie et de parenté. Dans la deuxième moitié du $\mathrm{XIX}^{\mathrm{e}}$ siècle, suivant la mutation de la chefferie vers une noblesse apanagée, la tenure foncière tongienne fut (légalement) transformée. Les nobles sont obligés de distribuer la plus grande partie de leurs domaines à ceux qui peuvent réclamer de la terre légalement. Cependant, on l'a vu, on est aujourd'hui dans un contexte de manque de terres. Alaric Maude 
avait déjà noté que le manque de terres « est aussi bien le résultat d'une commercialisation accrue de l'agriculture tongienne que de l'augmentation de la population » (1973: 174). Ici, deux autres points sont aussi d'importance: d'une part l'économie tongienne est toujours majoritairement agricole ; d'autre part, il est toujours illégal de vendre la terre. Toute la terre tongienne est contrôlée par les Tongiens.

En revanche, à Hawaii au milieu du XIX ${ }^{\mathrm{e}}$ siècle, selon $\mathrm{H}$. Trask, " la diplomatie de la canonnière [gunboat diplomacy] adoptée par les puissances occidentales et la duplicité missionnaire contre les chefs hawaiiens aboutirent à la transformation de la tenure foncière hawaiienne, autrefois utilisée communalement en propriété privée [...]; en 1888 , les trois quarts des terres cultivables étaient contrôlées par des haole ", c'est-àdire des étrangers blancs (1994: 16-18). Aujourd'hui, les Hawaiiens de souche ne contrôlent qu'une partie infime de terres. Le village de Keanae constitue une exception dans ce contexte car le pourcentage de propriétaires de terres hawaiiens y est relativement élevé. Ces villageois ont l'habitude de se voir comme des kama'aina, des résidents de longue date ou " des enfants de la terre ». Linnekin : "Ce sont des descendants des roturiers, les maka'aimana, et la plupart d'entre eux vivent à Keanae à cause d'une relation à quelqu'un à qui la terre appartenait autrefois » $(1985: 15)^{15}$. À Hawaii, contrairement à Tonga, la terre peut être achetée et vendue légalement, de même, l'agriculture hawaiienne n'est plus aujourd'hui l'activité économique principale, même si elle reste importante dans des villages comme Keanae. Comme nous l'avons déjà évoqué, le système de chefferie hawaiien a disparu dans le processus de colonisation. À Hawaii, l'unité domestique qui gère la terre correspond à la famille nucléaire. Ceci est formellement le cas à Tonga, depuis la transformation du système de tenure foncière au $\mathrm{XIX}^{\mathrm{e}}$ siècle mais, dans la pratique quotidienne de l'utilisation de la terre, les membres de la famille étendue jouent toujours un rôle crucial.

La quatrième caractéristique de la version tongienne de l'identité polynésienne correspond à une économie de subsistance et de don dans laquelle les cochons, les tubercules, les ressources halieutiques, le kava, les nattes et le tapa jouent un rôle prédominant. Aujourd'hui, l'agriculture tongienne est à la fois vivrière et commerciale, et son système économique repose sur la combinaison d'une économie de dons et d'une économie monétaire. Cathy Small a raison de souligner que : « villagers in Tonga could live a comfortable and enjoyable life without a substantial cash income ». Ce qui est possible parce que « (monetary) income makes much more of a difference when, as in industrial societies, one's entire existence depends on the purchasing power of money » (1997 : 27). Évidemment, ce n'est pas le cas dans les villages tongiens avec leur combinaison d'économies de subsistance et de dons. Écrivant sur son terrain dans les années 80 , Small observe que :

« In Tongan rural areas the subsistence economy was central to the quality of life. It was possible to grow staple food; to supplement this diet by fishing and by raising chickens and pigs; to produce building materials, flooring, bedding, cooking fuel, and many other necessities of life from the land and sea [...]. Although Tonga had become fully monetized, it did not develop the capitalism one might have envisioned. And although traditions in the village remained quite strong, many village institutions that passed for traditional were in fact radically changed from their precontact versions. In the village, kinship and capitalism, Tongan and Western, were thoroughly enmeshed, producing something [...] that was both and neither at the same time « (ibid., 27-28, 37).

C'est pour cela que " things were not what they appeared. Making money was just as much about fulfilling tradition as making tapa cloth was about acquiring capital and fund one's business » (ibid. 40). Pour Cathy Small — et je partage son point de vue - à Tonga, « kinship and capitalism, traditional and modern, Western and Tongan were thoroughly entwined rather than diametrically opposed » (ibid., 41), et je pense que ceci est toujours vrai aujourd'hui. Cependant, on doit ajouter certains éléments sur ce point. À Tonga, offrir des dons (le « gift complex ») est intimement lié à une "système de transfert de dons asymétrique qui rend apparent les relations de stratification » (Morgan, 1985 : 160) ${ }^{16}$. Les circuits de dons tongiens n'existent pas seulement entre parents, mais aussi entre roturiers (et membres de la classe moyenne) d'un côté, et royauté et noblesse de l'autre :

« Generosity to nobles, to those of higher rank, and to kin is highly valued [...]. Large amounts of cash now

15. Comme à Tonga, à Keanae, la distinction entre propriétaires terriens et gérants de la terre est importante : « Landholding in Keanae means not only having a legal share but actively managing the property — living on the land and using it. It is a well-established Hawaiian cultural principle that the right to use land is distinct from the right to dispose of it, so that landowner and landholder may be two different people » (Linnekin, $1985: 64-65$ ).

16. Dans les mots de l'auteur: "presentations to the sovereign and lords come as solicitary gifts and as periodic presentations in connection with visits and life-style ceremonies » (Morgan, $1985: 160$ ). 
change hands at weddings and funerals 'to cover expenses', which includes air fares for important guests or mourners, appropriate gifts, and clothing. Accordingly, it is not the rural cash-poor but more often their more prosperous, cash-rich, urban kin who are the mainstays of the traditional ceremonial system » (James, 1993: 217).

À Hawaii, la «transition d'un système redistributif polynésien vers une économie de marché est intimement liée au changement dans le fondement de l'autorité politique hawaiienne» (Linnekin, 1990 : 157). Aujourd'hui, parenté (hawaiienne) et capitalisme, tradition et modernité, polynésianité et occidentalité apparaissent diamétralement opposés dans les métaphores « intérieur » et « extérieur» (inside et outside), ce qui constitue « un excellent modèle de la société telle qu'elle est perçue par les Hawaiiens de Keanae » (Linnekin, 1985: 6). La métaphore fait référence d'une part aux éléments géographiques et sociaux comme par exemple campagne versus ville, hawaiien versus étranger, occident (la cité industrielle de Kahului) versus orient (l'ancienne ville hawaiienne de Hana), d'autre part, au système d'offrandes de dons dans la famille et parmi les amis, opposé aux transactions commerciales avec des personnes qui ne sont pas nécessairement des étrangers, mais qui ne sont ni (conçus comme de bons) membres de la famille ni (comme de vrais) amis : «le don opposé à la vente différencie leur communauté de l'extérieur » (ibid., 248). Bien que Keanae ait un pourcentage relativement élevé de propriétaires hawaiiens, un certain nombre d'entre eux cultivant le taro, un seul de ces cultivateurs le fait à plein temps: tous les autres, occupant des emplois salariés à plein temps, ne le font que temporairement ${ }^{17}$. Sur quarante-huit chefs de famille, huit travaillent à l'extérieur de Keanae dans les secteurs de la construction de bâtiments ou de routes et la moitié des familles dépendent $\mathrm{du}$ versement de pensions ou de la sécurité sociale. La plus grande partie de la nourriture dans les maisons de Keanae n'est pas produite mais achetée. Même dans les familles qui produisent du taro, le poi (purée de taro) est servi généralement accompagné de riz. Sur le marché ouvert, le poi est devenu une denrée de luxe (ibid., 34-35). La pêche est encore importante, mais davantage pour des raisons sociales que pour la subsistance. Chaque fois qu'une pêche dépasse la quantité ingurgitée au cours d'un repas, le surplus est stocké dans un grand congélateur qu'on trouve généralement dans chaque maison.
Ce surplus n'est pas destiné à une consommation future mais plutôt aux parents en visite (ibid., 56). Autrement dit, et contrairement à Tonga, à Hawaii les circuits d'offrandes et de dons sont limités à la famille et aux amis et ne s'étendent pas aux relations asymétriques entre roturiers et aristocrates.

À Hawaii, les boissons cérémonielles, comme le kava à Tonga, sont presque inexistantes. De même, en ce qui concerne le tapa et les nattes, un changement fondamental est apparu dès la deuxième moitié $\mathrm{du} \mathrm{XIX}^{\mathrm{e}}$ siècle, au moment où « le haut statut des chefs au lieu d'être marqué par les tapas et les nattes fines le fut par la soie, la vaisselle d'argent, et les services à thé [...]; l'enthousiasme des chefs pour les objets de luxe étrangers relégua les produits des femmes hawaiiennes à l'utilisation domestique et ordinaire » (Linnekin, $1990: 236$ ). Comme ailleurs en Polynésie, où « le travail des femmes n'est pas la production de la nourriture » (ibid., 37), « les anthropologues ont démontré que l'introduction des biens occidentaux eut un impact négatif sur le statut des femmes ", selon Linnekin, en particulier au travers du « remplacement des tissus faits à la main par des textiles importés » (ibid., 233 ; voir aussi Gailey, 1987 : 220-236). Pourtant, à Tonga, les produits du travail féminin - tapa et nattes - ont conservé un rôle social et symbolique éminent et les femmes de Tonga ont toujours, en tant que sœur, un statut valorisé. En effet, les structures sociales et le système de représentation au sein desquels ces éléments prennent leur sens continuent d'exister.

\section{Degrés différents de (ré)invention de la tradition}

Aujourd'hui, avec la perte du système de chefferie et de la structure d'autorité indigène «le cadre institutionnel de la société hawaiienne a disparu » (Linnekin, 1985 : 246). Tandis que les Tongiens ont conservé un appareil d'État souverain, « les Hawaiiens sont devenus historiquement le groupe le plus dépourvu de pouvoir de toute la société insulaire » (ibid., 6). Entre 1884 et 1890 , les Hawaiiens « devinrent une minorité numérique dans leur propre pays »; en 1898, avec l'annexion, « les Hawaiiens ont perdu leurs terres, leur mode de vie traditionnel, et leur autorité politique » (Linnekin, $1990: 226$ ). Au tournant du siècle, les Hawaiiens n'avaient plus ni de souveraineté politique ni d'autonomie économique, et leurs îles étaient confisquées par la force militaire américaine. Aujourd'hui, « les

17. Cultiver le taro est emblématique parce que « in Hawaiian genealogy [taro] is the kua'ana or elder sibling of the Hawaiian people» (H. Trask, $1994: 19)$. 
Hawaiiens ont été réduits à $20 \%$ de la population résidente » $(\mathrm{H}$. Trask, $1994: 18)$. À cela, on peut ajouter l'invasion annuelle de six millions de touristes ce qui, pour les Hawaiiens, « les condamne à une marginalisation dans leur propre pays » (ibid., 16). À Tonga, en revanche, « la relation entre identité, tradition culturelle et structure hiérarchique « est toujours très forte, et beaucoup de Tongiens s'inquiètent d'une transformation de la structure hiérarchique qui pourrait amener des changements de leur identité et leur tradition culturelle» (Morton, 1996 : 264).

Cette inquiétude peut être interprétée comme un signe de la force actuelle de la structure hiérarchique indigène de la société tongienne donc de convention - plutôt que de sa faiblesse. Le souci de la perte de l'identité et des repères culturels doit être évalué en regard du nouveau mouvement pour la démocratie, lequel représente une force d'égalisation sociale - au moins dans l'idéologie - visant à briser le monopole de l'aristocratie tongienne sur le pouvoir d'État. Une impulsion importante pour la genèse de ce mouvement fut la réduction d'une taxe sur les revenus des sociétés privées et l'introduction d'une nouvelle taxe sur la vente (l'équivalent de la TVA) en $1986{ }^{18}$. Rappelons, par ailleurs, que la force politique qui anime le mouvement pour la démocratie est la nouvelle classe moyenne tongienne. Les Hawaiiens, eux aussi, ont créé un mouvement politique radical, mais dans un contexte socio-politique complètement différent et avec d'autres finalités. À la fin des années 1960 apparaît le mouvement d'autonomie locale (ou nationale) à Hawaii : " mouvement urbain, attirant d'abord les jeunes métis hawaiiens qui ne parlaient pas hawaiien et dont la famille avait abandonné la vie rurale depuis longtemps » (Linnekin, 1985 : 9). À Tonga, la plupart des gens soit sont complètement intégrés dans la vie rurale, soit ont conservé un lien fort avec elle. Récemment, on a pu noter, toujours à Tonga, quelques inquiétudes au sujet de la perte de la « culture » :

«The recent emphasis on teaching Tongan language and culture can be seen in part as a response to the widespread concern that the younger Tongans are 'losing' their culture. Cultural workshops have been held for teachers, traditional singing and dancing is taught in schools, and a Tongan studies curriculum has been developed for high schools. The environmental science curriculum for primary schools has a social studies program that strongly emphasizes 'traditional culture' ") (Morton, 1996: 145).

Tous les Tongiens nés à Tonga — c'est-à-dire 98 à $99 \%$ de la population — parlent tongien comme langue maternelle. Dans le village de Keanae, sur l'île hawaiienne de Maui, où Jocelyn Linnekin a fait son terrain, « les résidents s'identifient comme hawaiiens, sans tenir compte de leur pourcentage d'ancestralité hawaiien [...] : [les villageois] ont l'habitude d'appeler 'Hawaiien' tout ceux qui ont une ancestralité partiellement hawaiienne pourvu que ce soit des amis ou des parents, des habitants de la campagne ou même qu'ils se comportent comme des Hawaiiens » (1985: 33). Il y a de nombreux mélanges ethniques ${ }^{19}$. Dans ce contexte, la tradition « est à la fois vécue et inventée [...], parce que les Hawaiiens ruraux se conforment à leurs propres attentes et à celles des autres de ce qui constitue la tradition » (ibid., 8 ; voir aussi Friedman, $1993: 765)^{20}$. Les instances les plus radicales du mouvement hawaiien revendiquent l'autonomie nationale ${ }^{21}$. Mililani Trask, avocate et leader politique, raconte comment, à la fin des années 1970, « un groupe de résidents indigènes formèrent Ho'ala Kanawai (Réveil de la Loi). Afin de renverser la politique de tutelle des États Unis, ils rassemblèrent et publièrent des informations historiques et politiques concernant les croyances indigènes [Native trusts] » (1994: 80-81). La souveraineté hawaiienne (ea), définie par le mouvement Ka Lahui Hawai'i (c'est-àdire: Pour la Souveraineté Indigène), doit consister en : (1) une croyance dans la spiritualité hawaiienne $(a k u a) ;(2)$ des traditions, une culture et un langage communs ; (3) un contrôle de la terre pour mettre en œuvre ces traditions ; (4) une autodétermination permettant de gérer la terre et les ressources naturelles ; (5) une autosubsistance économique permettant de se nour-

18. «Company and government agencies benefited, while poor people, farmers, and heavy consumers, including the rising middle class, lost » (Small, 1997 : 123).

19. "Hawaiians have a historically high rate of marriage to non-Hawaiians, and Keanae residents are no exception »; ethnicity, for Linnekin, being « a matter of orientation, identification, and personal choice » (Linnekin, $1985: 32$ ).

20. Linnekin spécifie que : «Tradition is a symbolic category, not an objectively definable body of artifacts and customs handed down from the past [...]. Every generation uses a model of the past to define itself, and this image is inevitably inverted to some extent because it is formulated in the current social context [...]. Tradition comprises that which is interpreted as being traditional in the present. The past is never received mechanically, without reflection and without alteration $»(1985: 241)$.

21. «The Hawaiian Movement evolved from a series of protests against land abuses, through various demonstrations and occupations to dramatize the exploitative conditions of Hawaiians, to assertions of Native forms of sovereignty based on indigenous birthrights to the land and sea » (H. Trask, 1994 : 19). 
rir, de s'habiller et d'abriter sa propre population (ibid., 84-85) 22 .

À Hawaii, la souveraineté indigène relève d'une revendication culturelle et politique. À Tonga, la souveraineté indigène - autorité politique suprême et indépendante - est un état de fait. Vu toutes ces différences, en particulier les quatre caractéristiques identifiées comme des composantes principales de l'identité polynésienne, il n'est pas surprenant que le mouvement pour la (ré-)invention de la tradition soit plus modéré à Tonga qu'à Hawaii. Bien que Tonga et Hawaii soient tous les deux impliqués simultanément dans la convention et l'invention - selon les termes de Roy Wagner - les Tongiens sont davantage concernés par la convention et les Hawaiiens par l'invention. Ceci étant, bien sûr, une question de degré.

Contrairement au concept dynamique de tradition vivante ( living tradition ») ${ }^{23}$, les traditions inventées sont «typiquement invariantes, utilisant des routines rituelles fixes ou des symboles stéréotypiques » (Jolly, $1992: 2$ ). Un de ces symboles stéréotypés pourrait être la devise héraldique tongienne 'Otua mo Tonga ko Hoku Tofi' $a$, c'est-à-dire «Dieu et Tonga Sont Mon Héritage ». Pour le formuler différemment: Dieu et Tonga sont conçus officiellement et représentés en termes (par exemple sur la monnaie nationale, le dollar tongien ou pa'anga) de tradition tongienne. Le dieu en question cependant n'est pas un dieu polynésien mais le dieu chrétien, introduit à Tonga par des missionnaires européens au $\mathrm{XIX}^{\mathrm{e}}$ siècle. Ces missionnaires britanniques et français ont fait leur travail avec tellement de succès que les Tongiens ont adopté les idées chrétiennes, les réorientant et en en faisant un des deux piliers officiels de leur propre tradition, exemple spécifique de l'invention de la tradition ${ }^{24}$. Il me paraît possible de dire que la transition socio-économique s'est développée et est toujours en train de se développer - de la même manière. Le capitalisme a ses origines en Europe et a été introduit à Tonga par les Européens et les Américains du Nord, et plus récem- ment, par les Japonais. Les idées et pratiques du capitalisme sont adoptées, réorientées et transformées par les Tongiens comme faisant partie de leur vie quotidienne. Les Tongiens habitant Tonga vivent bien entendu dans un seul monde. Ils peuvent en expérimenter les effets mais, habituellement, ils ne conçoivent ni ne conceptualisent les contradictions entre les différents systèmes capitaliste et polynésien, contradictions que peut révéler, en revanche, une analyse anthropologique de l'économie. Plutôt que d'une culture de compromis (« compromise culture»; Marcus, 1980), on pourrait parler de cet ensemble comme d'un bricolage culturel, comme une forme de pensée concrète (Lévi-Strauss, 1962 : 27). Ceci n'est pas spécifique à Tonga, c'est aussi vrai pour notre place dans notre propre société (occidentale ou autre). Ce qui est propre à Tonga, c'est le type même de contradiction et de bricolage culturel qui se trouve mis en œuvre. Des scientifiques sociaux et politiques peuvent devenir des maîtres dans l'analyse de la culture et de l'idéologie du pouvoir. Ceci n'enlève rien au pouvoir de l'idéologie et de la culture.

\section{BIBLIOGRAPHIE}

Abramson, Allen,. 1999. Sacred Cows of 'Development': The Ritual Incorporation of a Dairy Project in the Eastern Interior of Fiji (c. 1980-1997). Oceania 69 : 260-281.

BABADZAN, Alain (éd.), 1999. Les politiques de la tradition: Identités culturelles et identités nationales dans le Pacifique. Journal de la Société des Océanistes 109.

BARTH, Fredrick, 1966. Models of Social Organization. Occasional Paper 23. London: Royal Anthropological Institute of Great Britain and Ireland.

Bataille-Benguigui, Marie-Claire, 1994. Le côté de la mer: Quotidien et imaginaire aux îles Tonga, Polynésie occidentale. Collection Îles et Archipels no. 19. Bordeaux-Talence : Centre des Espaces Tropicaux

22. Souveraineté (ea) signifie une autorité politique suprême et indépendante ou, en termes hawaiiens avoir 'le mana de gouverner le pays' : " Before the coming of the haole [Whites], the idea of ea or sovereignty, of having political independence, was supported by the traditional 'aikapu religion. In traditional times, the Hawaiian polity was religious and Hawaiian religion, at the ali'i nui [high chiefly] level, was political. Therefore, ali'i nui were very religious, for without the approval from the akua, or gods, it was believed they had no long to rule. Ea was considered a gift from the akua, in particular from Ku, the $a k u a$ of war and of politics » (Kame'eleihiwa, $1994: 37$ ).

23. Smolicz: «A living tradition must be continually reshaped and revalued to meet the changing situation of the group. Such a dynamic concept allows each succeeding generation to evaluate various aspects of heritage in a new way: some parts may cease to interest, others take on a new importance, while valuations themselves can be reversed. This flexibility of tradition ensures its maintenance » $(1988: 390)$.

24. C'est à ce genre d'idéologie que Sahlins a fait allusion dans sa déclaration : « it was and still is a scandal to Christianized Polynesian people, one of the worst forms of 'idolatry' and reminders of their former 'darkness', that chiefs could be gods. For a long time now it has not been an easy topic for discussion, let alone a cherished treasure of collective memory » $(1995: 252)$. 
de l'Université Michel de Montaigne (Bordeaux III).

Belshaw, Cyril S., 1964. Under the Ivi Tree: Society and Economic Growth in Rural Fiji. London: Routledge and Kegan Paul.

—, 1965. Traditional Exchange and Modern Markets. Englewood Cliffs, N.J. : Prentice-Hall.

Benguigui, Georges, 1989. The Middle Classes in Tonga. Journal of the Polynesian Society 98 : 451463.

BIDESI, V.R., 1994. How 'the Other Half' Fishes: Accounting for Women in Fisheries in the Pacific. In 'Atu Emberson-Bain (éd.): Sustainable Development or Malignant Growth: Perspectives of Pacific Island Women, pp. 123-130. Suva, Mana Publications.

BiersaCK, Aletta, 1991. Kava'onau and the Tongan Chiefs. Journal of the Polynesian Society 100: 231268.

Boтt, Elizabeth, 1982. Tongan Society at the Time of Captain Cook's Visits. Memoir 44. Wellington, The Polynesian Society.

Brown, Richard and John ConNell, 1993. Entrepreneurs in the Emergent Economy: Migration, remittances and informal markets in the Kingdom of Tonga. Canberra: Economics Division, Research School of Pacific Studies, Australian National University.

Burrows, Edwin G., 1939. Breed and Border in Polynesia. American Anthropologist 41 : 1-21.

—, 1940. Culture Areas in Polynesia. Journal of the Polynesian Society 49 : 349-363.

CAMPBell, Ian C., 1992. Island Kingdom; Tonga Ancient and Modern. Christchurch: Canterbury University Press.

Douaire-Marsaudon, Françoise, 1996. Neither Black nor White: The Father's Sister in Tonga. Journal of the Polynesian Society 105 : 139-164.

—, 1998. Les premiers fruits: Parenté, identité sexuelle et pouvoirs en Polynésie. Collection Chemins de l'ethnologie. Paris : Éditions du CNRS et de la Maison des Sciences de l'Homme.

—, 2001. D'un sexe, l'autre: Le rituel du kava et la reproduction de l'identité masculine en Polynésie. L'Homme 157 : 7-34.

Dwyer, Peter, 1990. The pigs that Ate Gardens: A Human Ecology from Papua New Guinea. Ann Arbor: University of Michigan Press.

FisK, E.K. and D. Honeybone, 1971. Belshaw's 'Emergent Fijian Enterprise' After Ten Years. Pacific Viewpoint 12 : 123-140.

Friedman, Jonathan, 1993. Will the Real Hawaiian Please Stand: Anthropologists and Natives in the Global Struggle for Identity. In Paul van der Grijp and Toon van Meijl (eds) : Politics, Tradition and Change in the Pacific, pp. 737-767. Numéro spécial des Bijdragen tot de Taal-, Land-en Volkenkunde 149(3).
GaILEy, Christine W., 1981. Our History is Written in Our Mats: State Formation and the Status of Women in Tonga. PhD, Ann Arbor: University Microfim International.

—, 1987. Kinship to Kingship: Gender Hierarchy and State Formation in the Tonga Islands. Austin: University of Texas Press.

GIFFORD, Edward W., 1924. Tongan Myths and Tales. Bulletin 8. Honolulu: Bernice P. Bishop Museum.

—, 1929. Tongan Society. Bulletin 61. Honolulu: Bernice P. Bishop Museum.

GoDelier, Maurice, 1991. Transitions et subordinations au capitalisme. Paris : Maison des Sciences de l'Homme.

Goldman, Irving, 1970. Ancient Polynesian Society. Chicago and London: University of Chicago Press.

Hau'ofa, Epeli, 1994. Thy Kingdom Come: The Democratization of Aristocratic Tonga. The Contemporary Pacific 6 : 414-428.

HeLu, Futa, 1995. Brother/Sister and Gender Relations in Ancient and Modern Tonga. Journal de la Société des Océanistes 100-101 : 191-200.

—, 1999. Critical Essays: Cultural Perspectives from the South Seas. Canberra: The Journal of Pacific History.

Herda, Phyllis, 1988. The Transformation of the Traditional Tongan Polity: A Genealogical Consideration of Tonga's Past. PhD, Australian National University, Canberra.

Hobsbawm, Eric and Terence Ranger (eds), 1983. The Invention of Tradition. Cambridge: Cambridge University Press.

Hocart, A. M., 1915. The Chieftainship and the Sister's Son in the Pacific. American Anthropologist $17:$ 631-646.

Holmes, Lowell D. and Ellen Rhoads Holmes, 1992. Samoan Village: Then and Now. Second edition. Fort Worth: Holt, Rinehart and Winston.

Howard, Alan, 1996. Money, Sovereignty and Moral Authority on Rotuma. In Richard Feinberg and Karen Ann Watson-Gegeo (eds), Leadership and Change in the Western Pacific: Essays Presented to Sir Raymond Firth on the Occasion of His Ninetieth Birthday, pp. 205-238. London and Atlantic Highlands, N.J.: Athlone Press.

Howard, Alan and Robert Borofsky (eds), 1989. Developments in Polynesian Ethnology. Honolulu: University of Hawai'i Press.

JAMES, Kerry, 1993. Cutting the Ground from under Them? Commercialization, Cultivation, and Conservation in Tonga. The Contemporary Pacific $5(2): 215-242$.

-, 1994. The Pro-democracy Movement in Tonga. Pacific Affairs 67 : 242-263.

_, 1997. Reading the Leaves: The Role of Tongan Women's Traditional Wealth and Other 'Contraflows' in the Process of Modern Migration and Remittances. Pacific Studies 20 : 1-27. 
—, 1998. Analyzing the Emergent Middle Class — the 1990s. In Deryck Scarr, Niel Gunson and Jennifer Terrell (eds): Echoes of Pacific War, pp. 110-126. Canberra: Target Oceania.

Jolly, Margaret, 1992. Custom and the Way of the Land: Past and Present in Vanuatu and Fiji. Oceania 62: 330-354.

KAEPPLER, Adrienne, 1971. Rank in Tonga. Ethnology $10: 174-193$.

_, 1999. Kie Hingoa: Mats of Power, Rank, Prestige and History. Journal of the Polynesian Society 108: 168-232.

Kame’eleiHiwa, Lilikala, 1994. Ua Mau Ke Ea o Ka 'Aina i Ka Pono: The Concepts of Sovereignty and Religious Sanction of Correct Political Behavior. In Ulla Hasager and Jonathan Friedman (eds): Hawai'i: Return to Nationhood, pp. 34-43. Document 75. Copenhagen: International Work Group for Indigenous Affairs.

KeEsing, Roger and Robert Tonkinson (eds), 1982. Reinventing Traditional Culture: The Politics of Kastom in Island Melanesia. Numéro spécial de Mankind, 13(4).

LATUKefu, Sione, 1974. Church and State in Tonga: The Wesleyan Methodist Missionaries and Political Development 1822-1875. Canberra: Australian National University Press.

—, 1975. The Tongan Constitution: A Brief History to Celebrate its Centenary. Nuku'alofa: Government Printer.

-, 1994. The Pro-democracy Movement in Tonga. Journal of Pacific History 28 (3): 52-63.

_, 1997. The Impact of the British on the Tongan Traditional Concept of Justice and Law. In Hermann J. Hiery and John M. MacKenzie (eds): European Impact and Pacific Influence: British and german Colonial Policy in the Pacific Islands and the Indigenous Response, pp. 177-188. London and New York: Tauris.

Lawson, Stephane, 1996. Tradition versus Democracy in the South Pacific: Fiji, Tonga and Western Samoa. Cambridge: Cambridge University Press.

LEMONNIER, Pierre, 1993. Le porc comme substitut de vie: Formes de compensation et échanges en Nouvelle-Guinée. Social Anthropology 1a: 33-55.

LÉVI-Strauss, Claude, 1962. La pensée sauvage. Paris: Plon.

LINNEKIN, Jocelyn, 1985. Children of the Land: Exchange and Status in a Hawaiian Community. New Brunswick, New Jersey: Rutgers University Press.

_, 1990. Sacred Queens and Women of Consequence: Rank, Gender, and Colonialism in the Hawaiian Islands. Ann Arbor: University of Michigan Press.

MacPherson, Cluny, 1988. The Road to Power is a Chainsaw: Villages and Inno\$vation in Western Samoa. Pacific Studies 11(2): 1-24.
MAhinA, 'Okusitino, 1992. The Tongan Traditional History, Tala-e-Fonua: A Vernacular EcologyCentered Historico-Cultural Concept. PhD, Canberra: Australian National University.

Malm, Thomas, 1999. Shell Age Economics: Marine Gathering in the Kingdom of Tonga, Polynesia. Lund Monographs in Social Anthropology 8. Lund: Department of Sociology, Lund University.

Marcus, George E., 1980. The Nobility and the Chiefly Tradition in the Modern Kingdom of Tonga. Memoir 42. Wellington: The Polynesian Society.

Martin, John, 1817. An Account of the Natives of the Tonga Islands... Compiled and Arranged from the Extensive Communications of Mr. Mariner, Several Years Resident in Those Islands. 2 volumes. London. (consultée ici : l'édition facsimile éditée par Vava'u Press, Neiafu, 1981).

Matthews, E., 1995. Fishing for Answers: Women and Fisheries in the Pacific Islands. Suva: Women and Fisheries Networks.

Maude, Alaric, 1973. Land Shortage and Population Pressure in Tonga. In Harold G. Brookfield (ed.): The Pacific in Transiton: Geographical Perspectives on Adaptation and Change, pp. 163-186. London: Edward Arnold.

Morgan, Christopher, 1985. Competing Circuits in the Vava'u Social Economy. PhD, Canberra: Australian National University.

Morton, Helen, 1996. Becoming Tongan: An Ethnography of Childhood. Honolulu: University of Hawai'i Press.

O’Meara, Tim, 1990. Samoan Planters: Tradition and Economic Development in Polynesia. Fort Worth: Holt, Reinhart and Winston.

Oтто, Ton and Nicholas Thomas (eds), 1997. Narratives of Nation in the South Pacific. Amsterdam: Harwood Academic Publishers.

Perminow, Arne Aleksej, 1993. The Long Way Home: Dilemmas of Everyday Life in a Tongan Village. Oslo: Scandinavian University Press.

Powles, Charles Guy, 1990. The Early Accommodation of Traditional and English Law in Tonga. In Phyllis Herda, Jennifer Terrell and Niel Gunson (eds): Tongan Culture and History, pp. 145-169. Canberra: Department of Pacific and Southeast Asian History, Australian National University.

_, 1993. Tonga. In Michael A. Ntumy (réd.): South Pacific Islands Legal Systems, pp. 315-341. Honolulu: University of Hawai'i Press.

RADCLIFFE-Brown, A.R., 1924. The Mother's Brother in South Africa. South African Journal of Science 21 : 542-555.

Ramanlal, Soane, 1990. Private Sector Development in the Kingdom of Tonga. Honolulu: Pacific Islands Development Program, East-West Center.

RAPPAPORT, Roy, 1968. Pigs for the Ancestors: Ritual in the Ecology of a New Guinea People. New Haven: Yale University Press. 
RENSEL, Jan, 1994. For Love or Money? Interhousehold Exchange and the Economy of Rotuma. PhD, University of Hawai'i.

ReITER, F. 1907. Traditions tonguiennes. Anthropos 2 : 230-240.

RITTERBUSH, S. Deacon, 1988. Entrepreneurship in an Ascribed Status Society: The Kingdom of Tonga. In Te'o Fairbairn (ed.): Island Entrepreneurs: Problems and Performances in the Pacific, pp. 137-163. Honolulu: Pacific Islands Development Program, EastWest

Rogers, Garth, 1977. The Father's Sister is Black: A Consideration of Female Rank and Power in Tonga. Journal of the Polynesian Society 86 : 157181.

Rutherford, Noel. 1971. Shirley Baker and the King of Tonga. Melbourne: Oxford University Press.

SAHLIns, Marshall, 1958. Social Stratification in Polynesia. Seattle: American Ethnological Society.

—, 1995. How "Natives" Think: About Captain Cook, for Example. Chicago and London: University of Chicago Press.

SHore, Brad, 1989. Mana and Tapu. In Alan Howard and Robert Borofski (eds): Development in Polynesian Ethnology, pp. 137-173. Honolulu: University of Hawai'i Press.

Small, Cathy, 1997. Voyages: From Tongan Villages to American Suburbs. Ithaca and London: Cornell University Press.

Smolicz, J.J., 1988. Tradition, Core Values and Intercultural Development in Plural Societies. Ethnic and Racial Studies 11 : 387-410.

Tasman, Abel, 1643. Journael ofte Beschrijvinghe... aengaende de ontdeckinge van't onbekende Zuytland, in de jare anno 1642. In R. Posthumus Meyes (réd.): De reizen van Abel Janszoon Tasman en Franchoys de Visscher ter nadere ontdekking van het Zuidland, pp. 1-137. Edition facsimile. Tome 17. 's-Gravenhage: Linschooten Vereniging.

TCHERKÉZOFF, Serge, 1992. Les enfants de la terre aux îles Samoa: Tradition locale et "développement" importé. Etudes Rurales 127-128 : 15-40.

TIFfANy, Sharon, 1975. Entrepreneurship and Political Participation in Western Samoa: A Case Study. Oceania 46 : 85-106.

Trask, Haunani-Kay, 1994. Kupa'a 'Aina: Native Hawaiian Nationalism in Hawai'i. In Ulla Hasager and Jonathan Friedman (eds): Hawai' $i$ : Return to Nationhood, pp. 15-32. Document 75. Copenhagen: International Work Group for Indigenous Affairs.

Trask, Mililani B., 1994. The Politics of Oppression. In Ulla Hasager and Jonathan Friedman (eds): Hawai'i: Return to Nationhood, pp. 71-87. Docu- ment 75. Copenhagen: International Work Group for Indigenous Affairs.

TregeAr, Edward, 1891. The Maori-Polynesian Comparative Dictionary. Wellington: Lyon and Blair.

UrRY, John. 1993. Middle Class. In William Outhwaite and Tom Bottomore (eds): The Blackwell Dictionary of Twentieth-Century Social Thought, pp. 381-383. Oxford: Blackwell.

VAN DER GRIJP, Paul, 1992. Kerstening, staatsvorming en geweld op de Tonga Eilanden. Bijdragen tot de Taal-, Land-en Volkenkunde 148 : 31-58.

-, 1993a. After the Vanilla Harvest: Stains in the Tongan Land Tenure System. Journal of the Polynesian Society 102 : 233-253.

_, 1993b. Islanders of the South: Production, Kinship and Ideology in the Polynesian Kingdom of Tonga. Verhandelingen van het Koninklijk Instituut voor Taal-, Land- en Volkenkunde, tome 154. Leiden: KITLV Press.

-, 1993c. Christian Confrontations in Paradise: Catholic Proselytizing of a Protestant Mission in Oceania. Anthropos 88 : 135-152.

_, 1993d. The Making of a Modern Chiefdom State: The Case of Tonga. In Paul van der Grijp and Toon van Meijl (eds): Politics, Tradition and Change in the Pacific, pp. 661-672. Numéro spécial des Bijdragen tot de Taal-, Land-en Volkenkunde 149 (4).

_, 1993e. Women's Handicrafts and Men's Arts: The Production of Material Culture in the Polynesian Kingdom of Tonga. Journal de la Société des Océanistes 97 : 159-169.

_, 1997a. Brown Gold: Official Expectations and Local Assessment of the Tongan Vanilla Production. Journal de la Société des Océanistes 104 : 93-103.

-, 1997b. Leaders in Squash Export: Entrepreneurship and the Introduction of a New Cash Crop in Tonga. Pacific Studies 20(1) : 29-62.

_, 1999. Modernisation, mondialisation et identité polynésienne: Le modèle "MIRAB" dans le Pacifique. La Pensée 318 : 83-95.

VAn Der Grijp, Paul and Toon VAn MeiJl (eds), 1993. Politics, Tradition and Change in the Pacific. Numéro spécial des Bijdragen tot de Taal-, Land-en Volkenkunde 149, 4.

- 1994. European Imagery and Colonial History in the Pacific. Nijmegen Studies in Development and Cultural Change, volume 19. Saarbrücken: Breitenbach.

Wagner, Roy, 1975. The Invention of Culture. Englewood Cliffs, New Jersey: Prentice Hall.

Wolf, Eric, 1982. Europe and the People Without History. Berkeley et al. : University of California Press. 